TAO, Vol. 13, No. 2, 197-204, June 2002

NOTES AND CORRESPONDENCE

\title{
Source Parameters of Regional Earthquakes in Taiwan: January-December, 1998
}

\author{
Honn Kao ${ }^{1, *}$, Yu-Hua Liu ${ }^{1}$, Shuh-Chun Chen ${ }^{1}$, and Wen-Tzong Liang ${ }^{1}$
}

(Manuscript received 4 July 2001, in final form 20 February 2002)

\begin{abstract}
We report source parameters of 49 earthquakes that occurred between January and December, 1998, in the Taiwan region. The improved CMT inversion algorithm by Kao et al. (1998) is used to overcome the generally higher background noise as well as the heterogeneous velocity structure resulted from the complex tectonic interactions near Taiwan. To make the results more accessible and useful to the academic community, both the table that summarizes the reported source parameters and the complete set of inversion results are available electronically from the BATS worldwide web site at

http://bats.earth.sinica.edu.tw/CMT_Solutions/cmtF1998.html.
\end{abstract}

(Key words: Broadband Array in Taiwan for Seismology, earthquake source parameters, waveform inversion, Taiwan)

Successful development of the centroid-moment-tensor (CMT) inversion technique in the early 1980's has enabled the systematic determination of source parameters for global large and moderately-sized earthquakes (e.g., Dziewonski et al. 1981; Kawakatsu et al. 1995; Sipkin 1982). Later, the CMT inversion technique was successfully applied to regional earthquakes because of the increasing knowledge in detailed velocity structures on a regional scale and the establishment of regional broadband networks (e.g., Dreger and Helmberger 1993; Fan and Wallace 1995; Lay et al. 1994; Thio and Kanamori 1995; Zhao and Helmberger 1994). Consequently, routine report of CMT solutions for smaller, regional earthquakes becomes standard practice for many regional broadband seismographic networks (e.g., Zhu and Helmberger 1996; Pasysnos et al. 1996).

Establishment of the "Broadband Array in Taiwan for Seismology (BATS)" has enabled the systematic determination of reliable source parameters for regional earthquakes in Taiwan through CMT technique (Kao et al. 1998). The network began its test operation in late 1994 (Fig. 1). A brief description of BATS configuration and operation can be found in Kao et al.

\footnotetext{
${ }^{1}$ Institute of Earth Sciences, Academia Sinica, Taipei, Taiwan, ROC

${ }^{*}$ Corresponding author address: Dr. Honn Kao, Institute of Earth Sciences, Academia Sinica, P.O. Box 1-55, Nankang, Taipei, Taiwan, ROC; E-mail: kao@earth.sinica.edu.tw
} 


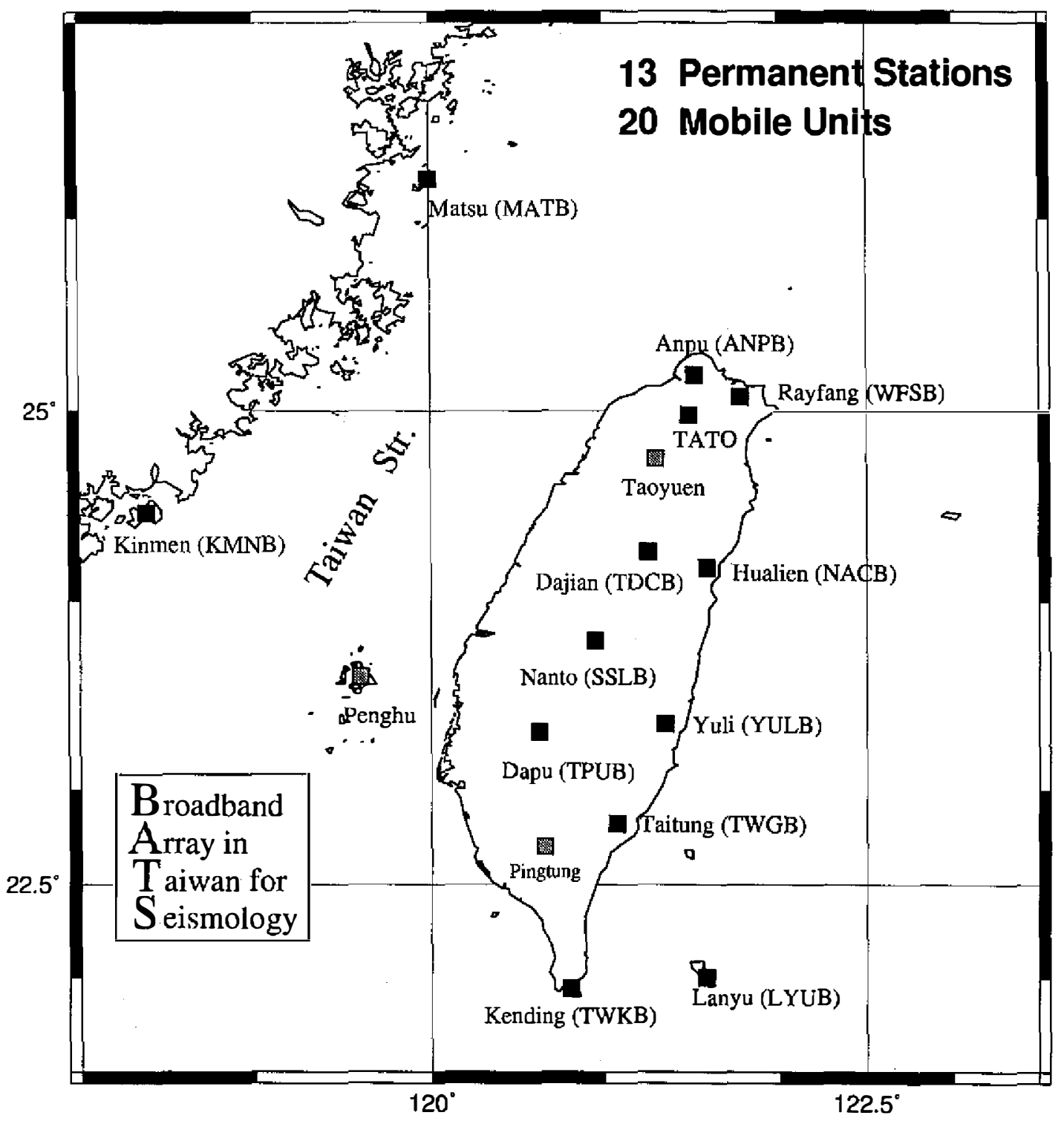

Fig. 1. Map of the "Broadband Array in Taiwan for Seismology (BATS)". Solid and gray squares show stations currently in operation and under construction, respectively. In addition to the permanent stations, BATS includes 17 portable stations that can be deployed for specific research tasks. 
$(1988,2001)$ and Kao and Jian $(1999,2001)$. The Data Management Center of the Institute of Earth Sciences (IES-DMC), Academia Sinica is responsible for BATS data archiving and distribution. Meanwhile, one copy of BATS data is contributed to the Data Management Center of the Incorporated Research Institutions for Seismology (DMC, IRIS) for the same purpose. Interested readers can obtain updated information at BATS world-wide web site (http://bats. earth.sinica.edu.tw).

Our CMT algorithm begins with a background-noise evaluation that determines the frequency band used in the inversion. The cut-off frequency of the lower comer is usually at $\sim 0$. $02 \mathrm{~Hz}$, which is determined by comparing the power spectra of waveform windows $300 \mathrm{~s}$ before and after the $P$ first arrival (Kao et al. 1998). We also utilize a two-step procedure to allow different velocity models for different station-source pairs. This is to mimic the effect of heterogeneous velocity structures in the Taiwan region. For more technical details, readers are referred to our previous reports (Kao et al. 1998; Kao and Jian 1999).

To characterize the quality of inversion, each inversion result is classified by a combination of a letter (A-F) and a digit (1-4) depending on two parameters: the waveform misfit (E) and the amount of CLVD component $(\varepsilon)$, respectively. The derivations of $\mathrm{E}$ and $\mathrm{e}$ are presented in our previous reports (Kao et al. 1998; Kao and Jian 1999) and listed in Table 1.

Following the criteria defined in Kao and Jian (1999), we report source parameters only if they meet the following criteria: (1) 3-component waveforms from at least three stations are used in the inversion, and (2) the quality of inversion must be higher than C4. A total of 49 earthquakes that satisfy these conditions are listed in Table 2 . The corresponding best doublecouple solutions and focal depths are plotted in Fig. 2.

Due to the practical concern, the inversion results are presented as an electronic appendix to this report. Interested readers can download the complete set from BATS world-wide web site at http://bats.earth.sinica.edu.tw/CMT_Solutions/cmtF1998.html. To make the results more accessible and useful to the academic community, Table 2 as well as the tables in our previous

Table 1. Quality classification of inversion results.

\begin{tabular}{cc} 
Class & Criteria \\
\hline & Average Waveform Misfit (E) \\
A & $0 \leq \mathrm{E}<0.3$ \\
B & $0.3<\mathrm{E} \leq 0.5$ \\
C & $0.5<\mathrm{E} \leq 0.7$ \\
D & $0.7<\mathrm{E} \leq 0.9$ \\
E & $0.9<\mathrm{E} \leq 1.1$ \\
F & E $>1.1$ \\
& CLVD component $(\varepsilon)$ \\
1 & $\varepsilon \leq 0.1$ \\
2 & $0.1<\varepsilon \leq 0.25$ \\
3 & $0.25<\varepsilon \leq 0.4$ \\
4 & $\varepsilon>0.4$ \\
\hline
\end{tabular}


Table 2. Source parameters of studied earthquakes.

\begin{tabular}{|c|c|c|c|c|c|c|c|c|c|c|c|c|c|c|c|c|c|}
\hline No & Origin Time $^{1}$ & at. ${ }^{1}$ & Long. ${ }^{1}$ & Dep. & $\mathbf{M}_{\mathbf{x} x}^{2}$ & $\mathrm{M}_{y y}{ }^{2}$ & $\mathrm{M}_{z z}^{2}$ & $M_{x y}^{2}$ & $\mathrm{M}_{\mathrm{xz}}{ }^{2}$ & $\mathrm{M}_{\mathrm{yz}^{2}}{ }^{2}$ & $\mathrm{M}_{\mathrm{w}}{ }^{2}$ & Strike $^{3}$ & $\mathrm{Dip}^{3}$ & $\mathrm{Rake}^{3}$ & $E^{4}$ & $\varepsilon^{4}$ & Class \\
\hline 1 & $98 / 01 / 18 / 19: 56: 51.7$ & 22.73 & $\$ 21.09$ & 15 & -11.88 & -17.73 & 45.08 & 26.36 & 28.87 & -67.13 & 5.22 & 239.98 & 17.60 & 123.05 & 0.547 & 5.93 & $\mathrm{Cl}$ \\
\hline 2 & 98/01/20/23:29:38.9 & 22.69 & 121.08 & 18 & -0.44 & -3.80 & 6.19 & 2.32 & 1.50 & -7.59 & 4.59 & 218.80 & 19.22 & 113.21 & 0.552 & $3.81^{\circ}$ & SI \\
\hline 3 & 98/02/07/07:20:52.1 & 23.63 & 121.50 & 24 & 0.17 & -0.95 & 0.59 & 0.49 & -0.65 & 0.47 & 4.00 & 348.92 & 34.72 & 36.87 & 0.487 & 27.30 & B3 \\
\hline 4 & $98 / 02 / 24 / 06: 45: 31.2$ & 24.41 & 121.69 & 27 & -0.29 & -0.28 & 0.18 & 1.40 & -0.02 & -1.80 & 4.18 & 266.87 & 37.73 & 171.88 & 0.580 & 0.00 & $\mathrm{Cl}$ \\
\hline 5 & $98 / 02 / 24 / 06: 59: 45.3$ & 24.40 & 121.69 & 30 & -0.30 & 0.38 & -0.15 & 0.85 & 0.02 & -0.52 & 3.95 & 280.69 & 59.96 & -177.70 & 0.508 & 25.73 & $\mathrm{C} 3$ \\
\hline 6 & 98/03/11/06:00:08.1 & 22.36 & 122.30 & 30 & 5.60 & -4.15 & -1.74 & -0.47 & 2.39 & -1.08 & .4 .44 & 133.09 & 61.17 & -4.60 & 0.551 & 1.09 & $\mathrm{C} 4$ \\
\hline 7 & 98/03/11/17:21:54.9 & 22.44 & 122.26 & 30 & 33.38 & -39.25 & -1.34 & 2.99 & 1.86 & 6.36 & 4.98 & 47.69 & 81.03 & -175.99 & 0.544 & 10.24 & $\mathrm{C} 2$ \\
\hline 8 & 98/04/19/10:06:27.0 & 24.39 & 121.71 & 30 & -0.20 & 0.20 & -0.10 & 0.80 & 0.09 & -1.36 & 4.07 & 277.16 & 31.15 & -176.99 & 0.522 & 7.23 & $\mathrm{Cl}$ \\
\hline 9 & 98/04/28/07:09:00.9 & 23.97 & 121.69 & 24 & -0.10 & 0.11 & 0.07 & 0.07 & 0.44 & -0.37 & 3.78 & 298.70 & 9.86 & 159.97 & 0.544 & 20.50 & $\mathrm{C} 2$ \\
\hline 10 & 98/05/02/00:05:53.0 & 21.72 & 121.10 & 24 & 2.07 & $-1 \mathrm{I} .42$ & 6.20 & -0.42 & 3.49 & 0.30 & 4.60 & 24.66 & 50.81 & 129.61 & 0.629 & 22.24 & $\mathrm{C} 2$ \\
\hline 11 & 98/05/02/05:37:42.4 & 24.56 & 122.56 & 84 & -7.85 & 1.62 & 6.46 & 4.19 & 17.12 & 6.90 . & 4.81 & 221.42 & 20.03 & 26.23 & 0.467 & 16.84 & B2 \\
\hline 12 & 98/05/09/20:53:39.0 & 24.82 & 121.91 & 72 & 0.63 & -6.18 & 5.01 & -3.09 & 11.05 & 6.02 & 4.70 & 5.75 & 21.12 & 149.56 & 0.434 & 14.93 & B2 \\
\hline 13 & 98/05/15/08:50:28.5 & 23.96 & 121.72 & 39 & 1.60 & -0.95 & -0.74 & -1.16 & -0.87 & 1.34 & 4.19 & 290.74 & 43.45 & -15.53 & 0.475 & 32.96 & B3 \\
\hline 14 & $98 / 05 / 17 / 16: 36: 01.2$ & 24.08 & 121.53 & 27 & -0.59 & -0.34 & 0.80 & 0.76 & 0.04 & 0.49 & 3.98 & 75.95 & 43.91 & 131.69 & 0.529 & 22.97 & $\mathrm{C} 2$ \\
\hline 15 & 98/05/30/06:22:38.3 & 24.27 & 122.13 & 57 & 2.01 & -8.81 & 6.33 & 3.00 & 3.99 & 4.75 & 4.62 & 43.77 & 41.63 & 141.27 & 0.429 & 4.02 & $\mathrm{~B} 1$ \\
\hline 16 & 98/07/06/1 I:33:54.2 & 24.35 & 122.87 & 39 & -1.69 & 1.37 & 0.26 & 0.13 & 1.43 & -1.2 & 4.20 & 312.14 & 38.38 & 173.64 & 0.489 & $18.8 \mathrm{I}$ & B2 \\
\hline 17 & $98 / 07 / 11 / 18: 23: 14.9$ & 24.37 & 122.04 & 21 & -0.04 & 0.02 & 0.16 & -0.62 & 0.13 & -0.47 & 3.87 & 92.98 & 54.04 & 11.56 & 0.483 & 21.31 & B2 \\
\hline 18 & 98/07/17/04:51:15.0 & 23.50 & 120.66 & 12 & 79.84 & -318.13 & 169.46 & 82.57 & -193.35 & 197.70 & 5.66 & 339.58 & 32.00 & 35.57 & 0.559 & 21.23 & $\mathrm{C} 2$ \\
\hline 19 & $98 / 07 / 17 / 18: 44: 39.9$ & 24.06 & 121.69 & 15 & -1.56 & -5.04 & 5.03 & 2.30 & 3.64 & -4.09 & 4.53 & 19 & 2 & 70.96 & 5 & 2.09 & $\mathrm{~B} 1$ \\
\hline 20 & 98/07/18/17:02:39.7 & 23.51 & 120.69 & 12 & 1.33 & -0.75 & -0.22 & 1.09 & -2.43 & 1.92 & 4.30 & 327.57 & 25.73 & -3.64 & 0.503 & 40.84 & $\mathrm{C} 4$ \\
\hline 21 & 98/07/19/17:58:31.9 & 23.53 & 1167 & 36 & 0.17 & -3.38 & 3.07 & 1 & 0.07 & -1.4 & 4.32 & 201.78 & 33.89 & 100.27 & 0.453 & 24.12 & B2 \\
\hline 22 & $98 / 07 / 20 / 16: 35: 24.9$ & 24.08 & 122.10 & 21 & -3.35 & -1.79 & 6.49 & 2.72 & 2.56 & -4.16 & 4.53 & 243.97 & 27.18 & 111.43 & 0.581 & 12.33 & $\mathrm{C} 2$ \\
\hline 23 & 98/07/24/18:44:03.0 & 21.63 & 121.84 & 12 & 467.62 & -994.06 & 360.10 & 46.15 & -409.80 & -468.71 & 5.94 & 220.10 & 44.59 & 157.02 & 0.557 & 25.66 & $\mathrm{C} 3$ \\
\hline 24 & 98/07/30/08:15:41.1 & 22.93 & 121.41 & 15 & 0.16 & -0.58 & 0.49 & 0.18 & -0.32 & 0.13 & 3.82 & 345.61 & 42.72 & 44.42 & 0.487 & 4.75 & Bl \\
\hline 25 & $98 / 07 / 31 / 09: 23: 26.3$ & 24.76 & 122.87 & 111 & 3.65 & -2.67 & -0.78 & 9.69 & -5.13 & 1.12 & 4.64 & 350.24 & 62.60 & -3.79 & 0.495 & 5.82 & Bl \\
\hline 26 & $98 / 08 / 11 / 02: 07: 49.8$ & 24.85 & 123.34 & 129 & -15.65 & 9.09 & 7.09 & 48.06 & -58.38 & -6.51 & 5.19 & 8.73 & $40.0 \mathrm{I}$ & 5.07 & 0.464 & 4.38 & $\mathrm{~B} 1$ \\
\hline
\end{tabular}


(Table 2. continued)

\begin{tabular}{|c|c|c|c|c|c|c|c|c|c|c|c|c|c|c|c|c|c|}
\hline 7 & 98/08/13/01:05:31.3 & 24.47 & 122.97 & 81 & -0.63 & 0.66 & -0.08 & 0.20 & 0.58 & 0.43 & 3.93 & 215.64 & 42.96 & -3.45 & 0.396 & 3.59 & $\mathrm{~B} 1$ \\
\hline 8 & $98 / 08 / 15 / 00: 15: 43.3$ & 24.39 & 122.77 & 24 & -0.76 & 0.07 & 0.92 & 0.27 & 0.57 & -0.31 & 3.96 & 262.10 & 27.57 & 104.25 & 0.488 & 9.91 & $\mathrm{Bl}$ \\
\hline 29 & 98/08/16/11:13:03.8 & 23.20 & 120.53 & 12 & 2.09 & -3.79 & 2.85 & 0.84 & .99 & 0.14 & 4.36 & 5.00 & 54.01 & 37.45 & 494 & 8.76 & 31 \\
\hline & $98 / 08 / 25 / 02: 49: 31.2$ & 24.60 & 122.53 & 15 & 5.64 & -6.86 & -0.02 & 2.77 & -1.24 & -0.55 & 4.50 & 236.42 & 80.32 & 173.37 & 0.590 & 3.91 & $\mathrm{Cl}$ \\
\hline & $98 / 09 / 04 / 10: 40: 50.6$ & 23.91 & 121.58 & 18 & -2.99 & 0.30 & 2.03 & 4.77 & 2.69 & 1.64 & 4.47 & 195.57 & 57.28 & 22.28 & 0.509 & 7.03 & $\mathrm{C} 1$ \\
\hline 27 & 98/09/09/17:54:00.8 & 23.10 & 121.53 & 36 & 0.66 & -0.47 & -0.16 & 0.21 & 0.20 & -0.21 & 3.83 & 142.63 & 62.44 & -13.07 & .435 & 9.60 & 82 \\
\hline 33 & 98/09/10/06:46:06.4 & 22.64 & 120.66 & 15 & 0.21 & -3.06 & 2.68 & -0.32 & -1.31 & 0.93 & 4.28 & 333.02 & 41.66 & 54.04 & 0.484 & 17.21 & B2 \\
\hline 34 & 98/09/12/06:53:08.5 & 24.43 & 122.61 & 42 & 0.79 & -1.63 & 0.86 & -0.14 & -0.92 & -0.27 & 4.09 & 1.30 & 52.28 & 148.71 & 0.478 & 5.91 & B1 \\
\hline 35 & 98/09/13/05:34:48.3 & 24.24 & 123.01 & 33 & 31.67 & .46 .79 & 21.57 & -10.96 & -28.91 & -1.92 & 5.09 & 209.81 & 58.94 & 153.61 & 0.474 & 12.20 & B2 \\
\hline 36 & 98/09/16/04:56:11.2 & 23.84 & 120.84 & 27 & -0.11 & -0.34 & 0.43 & 0.40 & -0.23 & -0.32 & 3.83 & 246.78 & 48.40 & 139.69 & 0.368 & 19.27 & B2 \\
\hline 37 & 98/09/2 1/16:29:37.3 & 23.94 & 121.55 & 15 & -0.76 & -0.26 & 0.68 & 0.98 & 0.52 & 0.58 & 4.05 & 198.58 & 56.82 & $37.3 \mathrm{I}$ & 0.477 & 10.87 & B2 \\
\hline 20 & 98/09/27/17:02:22.0 & 24.48 & 122.42 & 51 & -0.23 & -0.77 & 1.22 & 0.88 & 0.08 & 0.57 & 4.05 & 57.93 & 40.46 & 123.59 & 0.370 & 24.87 & B2 \\
\hline 39 & 98/09/28/08:57:19.9 & 21.09 & 122.11 & 36 & -5.71 & -0.10 & 3.80 & -3.67 & -5.91 & -12.64 & 4.73 & 86.75 & 18.85 & 26.91 & 0.619 & 9.16 & $\mathrm{Cl}$ \\
\hline 40 & $98 / 10 / 09 / 12: 52: 51.5$ & 22.12 & 121.65 & 27 & 5.10 & -22.81 & 16.20 & 4.48 & 4.12 & 3.52 & 4.82 & 28.85 & 44.60 & 119.75 & 0.397 & 39.52 & B3 \\
\hline 41 & $98 / 10 / 11 / 04: 56: 34.8$ & 23.89 & 121.71 & 45 & 0.44 & -0.31 & -0.15 & 0.23 & -0.26 & 0.21 & 3.77 & 326.25 & 51.75 & -14.70 & 0.463 & 3.35 & $\mathrm{BI}$ \\
\hline 42 & 98/10/13/16:54:18.0 & 21.86 & 121.50 & 24 & 1.42 & -0.16 & -0.97 & 0.70 & -0.22 & -1.01 & 4.10 & 260.85 & 42.88 & -142.39 & 0.477 & 4.43 & B] \\
\hline J & $98 / 10 / 17 / 23: 11: 09.7$ & 24.82 & 122.01 & 27 & 2.10 & -4.02 & 2.79 & -2.06 & 3.14 & -3.20 & 4.47 & 129.76 & 42.64 & 24.74 & 0.536 & 28.46 & $\mathrm{C} 3$ \\
\hline 44 & 98/1 1/03/07:06:37.5 & 22.15 & 121.15 & 36 & 1.40 & -4.84 & -0.35 & -13.40 & -5.74 & 2.97 & 4.73 & 184.14 & 70.21 & 167.02 & 0.361 & 20.66 & B2 \\
\hline 45 & $98 / 11 / 17 / 22: 27: 32.5$ & 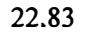 & 120.79 & 24 & 0.0 & -00.10 & 68.44 & -33.69 & -52.29 & 80.0 & 5.2 & 0 & 2 & 1 & 38 & 13.39 & 2 \\
\hline 40 & $98 / 11 / 19 / 10: 30: 02.2$ & 23.79 & 121.77 & 48 & -2.07 & 1.18 & 0.58 & 2.53 & -1.15 & -1.38 & 4.30 & 20.65 & 64.78 & 18.69 & 0.377 & 17.37 & B2 \\
\hline 47 & 98/11/26/22:12:19.3 & 25.06 & 122.51 & 159 & -0.09 & 10.05 & -9.99 & 3.49 & -2.50 & 3.60 & 4.64 & 144.99 & 40.72 & -118.32 & 0.318 & 0.63 & Bl \\
\hline 48 & 98/12/14/00:59:03.3 & 24.29 & 122.16 & 48 & 0.57 & -4.37 & 3.26 & 1.95 & 1.41 & 3.07 & 4.43 & 47.24 & 37.19 & 138.90 & 0.351 & 6.78 & B1 \\
\hline 49 & 98/12/22/01:49:06.9 & 24.25 & 122.58 & 60 & 2.19 & -1.73 & -0.75 & 2.89 & 0.91 & 5.99 & 4.50 & 79.08 & 30.37 & -174.79 & 0.366 & 23.08 & 2 \\
\hline
\end{tabular}

${ }^{\mathrm{J}}$ Origin time (Year/Month/Day/hr:min:sec) and epicentral locations $\left({ }^{\circ} \mathrm{N},{ }^{\circ} \mathrm{E}\right)$ are reported by the Seismology Center, Central Weather Bureau, Taiwan.

${ }^{2} \mathrm{X}, \mathrm{Y}, \mathrm{Z}$ point to north, east, and vertically down, respectively. All are in the unit of $1 \times 10^{15} \mathrm{Nt} \mathrm{m}$.

${ }^{3}$ Estimated best double-couple solutions in degrees.

${ }^{4} \mathrm{E}$ and $\varepsilon$ are defined by equations (2) and (1), respectively. $\varepsilon$ is expressed in percent (\%). 


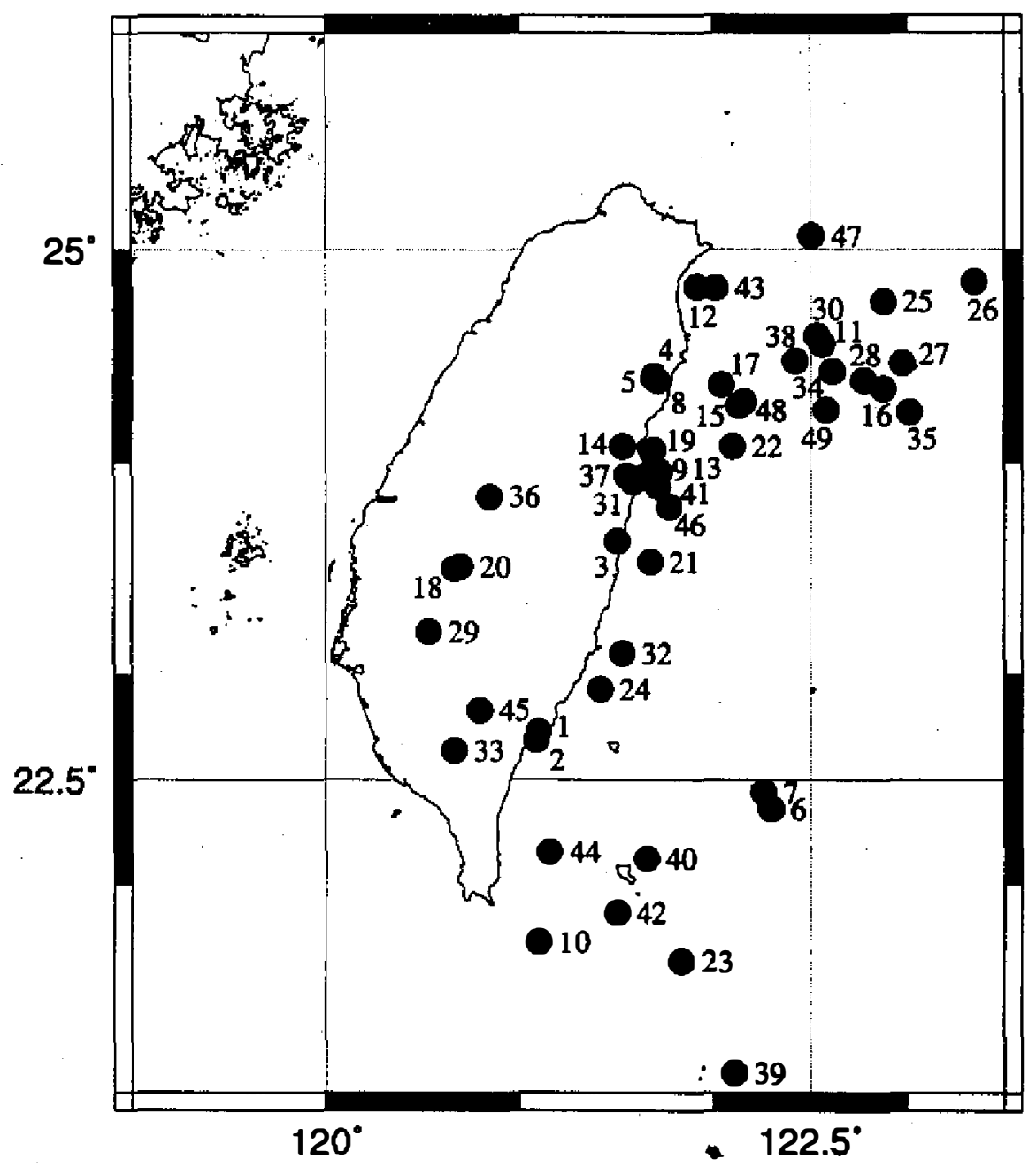

Fig. 2. Moment-tensor inversion results. (a) Map shows the epicenters of 49 earthquakes presented in this study. Numbers are according to Table 2. (b) Corresponding best double-couple solutions. The first number above each fault plane solution is the event number. The number in parenthesis is the best focal depth.

reports showing source parameters of earthquakes between 1995 and 1997 are also available in our web site (http://bats.earth.sinica.edu.tw/CMT_Solutions).

Acknowledgments Technical staff at IES led by Chun-Chi Liu assumes the primary responsibility for maintaining BATS. This research was partially supported by the National Science Council; Republic of China, under grants NSC89-2921-M-001-012-EAF and NSC90-2119M-001-011. 

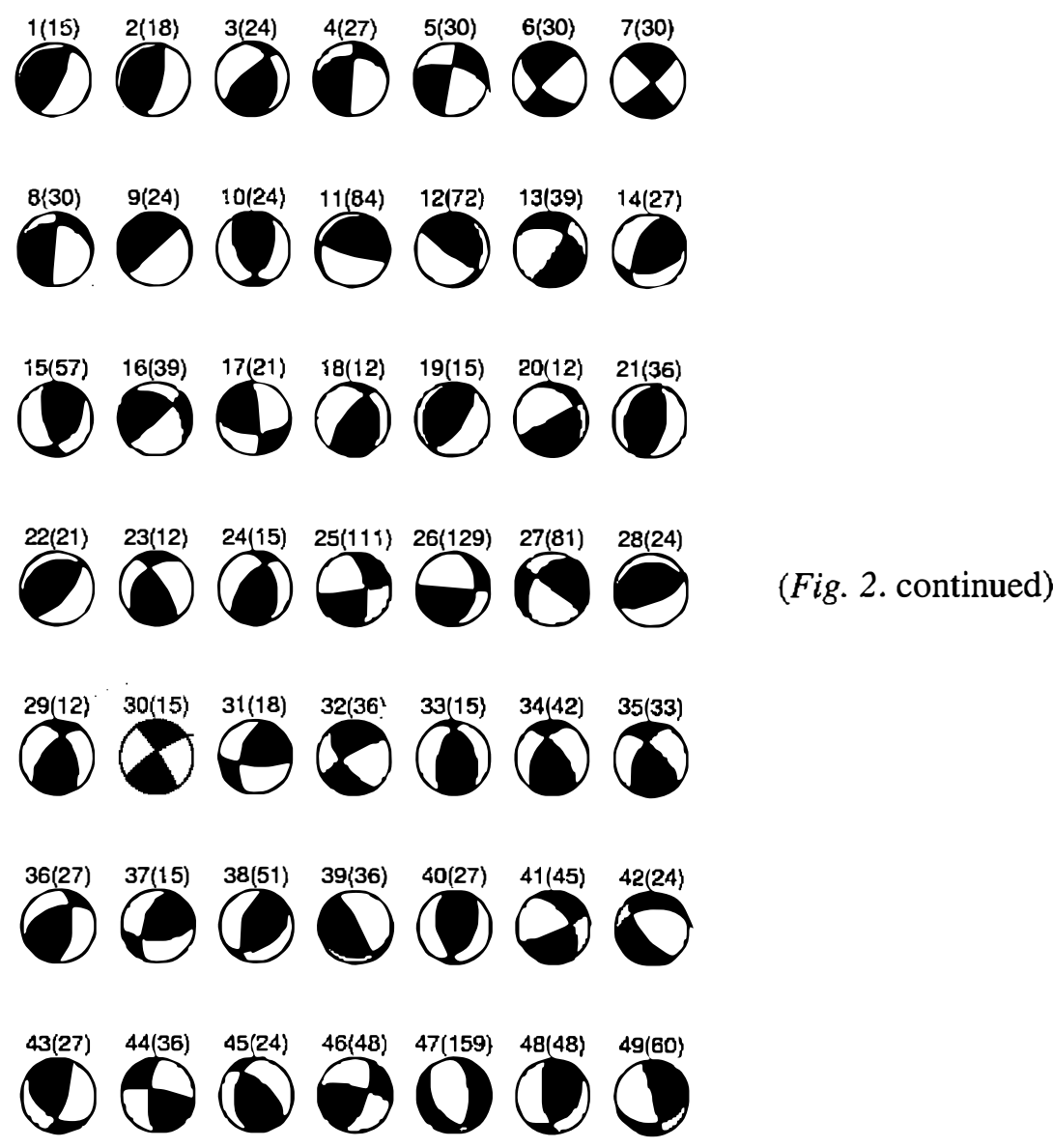

\section{REFERENCES}

Dreger, D. S., and D. V. Helmberger, 1993: Determination of source parameters at regional distances with three-component sparse network data. J. Geophys. Res., 98, 8107-8125.

Dziewonski, A. M., T.-A. Chou, and J. H. Woodhouse, 1981: Determination of earthquake source parameters from waveform data for studies of global and regional seismicity. $J$. Geophys. Res., 86, 2825-2852.

Fan, G., and T. Wallace, 1995: Focal mechanism of a recent event in South Africa: A study using a sparse very broadband network. Seismol. Res. Lett., 66, 13-18.

Kao, H., and P.-R. Jian, 1999: Source parameters of regional earthquakes in Taiwan: July 1995-December 1996. TAO, 10, 585-604.

Kao, H., P.-R. Jian, K.-F. Ma, B.-S. Huang, and C.-C. Liu, 1998: Moment-tensor inversion for offshore earthquakes east of Taiwan and their implications to regional collision. Geophys. Res. Lett., 25, 3619-3622. 
Kao, H., and P.-R. Jian, 2001: Seismogenic patterns in the Taiwan region: Insights from source parameter inversion of BATS data. Tectonophysics, 333, 179-198.

Kao, H., Y.-H. Liu, and P.-R. Jian, 2001: Source parameters of regional earthquakes in Taiwan: January-December 1997. TAO, 12, 431-439.

Kawakatsu, H., 1995: Automated near-realtime CMT inversion. Geophys. Res. Lett., 22, 25692572.

Lay, T., J. Ritsema, C. J. Ammon, and T. Wallace, 1994: Rapid source-mechanism analysis of the April 29, 1993 Cataract Creek $\left(M_{w}=5.3\right)$, northern Arizona earthquake. Bull. Seismol. Soc. Am., 84, 451-457.

Pasyanos, M. E., D. S. Dreger, and B. Romanowicz, 1996: Toward real-time estimation of regional moment tensors. Bull. Seismol. Soc. Am., 86, 1255 1269.

Sipkin, S. A., 1982: Estimation of earthquake source parameters by the inversion of waveform data: synthetic waveforms. Phys. Earth Planet. Inter., 30, 242-259.

Thio, H. K., and H. Kanamori, 1995: Moment-tensor inversions for local earthquakes using surface waves recorded at TERRAscope. Bull. Seismol. Soc. Am., 85, 1021-1038.

Zhao, L.-S., and D. V. Helmberger, 1994: Source estimation from broadband regional seismograms. Bull Seismol. Soc. Am., 84, 91-104.

Zhu, L., and D. V. Helmberger, 1996: Advancement in source estimation techniques using broadband regional seismograms. Bull. Seismol. Soc. Am., 86, 1634-1641. 